\title{
Selective intraarterial gene delivery into a canine meningioma
}

\section{Anne E. Chauvet, D.V.M., Prabhakar P. Kesava, M.D., Chern Sing Goh, B.S., and Behnam Badie, M.D.}

Department of Medical Sciences, School of Veterinary Medicine, and Department of Radiology and Neuro-oncology Laboratory, Department of Neurological Surgery, School of Medicine, University of Wisconsin, Madison, Wisconsin

Object. The goal of this study was to evaluate gene delivery to a benign brain tumor.

Methods. A recombinant adenovirus vector bearing the Escherichia coli ß-galactosidase reporter gene was selectively injected into the vascular supply of a spontaneously occurring canine olfactory groove meningioma. The tumor and a small amount of peritumoral brain tissue were removed 5 days after viral injection and stained with X-Gal to assess gene delivery. The authors noted significant B-galactosidase gene expression by the tumor, but not by surrounding brain tissue. No obvious viral-related cytotoxicity was noted.

Conclusions. The authors found that meningiomas can be successfully transduced by adenovirus vectors by using endovascular techniques.

\section{Key Words * gene therapy * adenoviral vector * meningioma * intracarotid * dog}

Malignant brain tumors are ideal candidates for gene therapy because they respond poorly to current treatment modalities, recur only locally, and rarely metastasize to other organs. Although different viral vectors are being evaluated for gene delivery into these neoplasms, retrovirus-mediated suicide gene therapy has been most extensively studied both in the laboratory and clinical settings.[3]

Replication-defective adenoviruses also have shown promise as excellent vectors for gene delivery into a variety of organs including the central nervous system (CNS).[6] As opposed to retroviruses, adenoviruses are capable of high-efficiency gene delivery to both mitotic and nonmitotic cells. They also persist extrachromosomally, thereby minimizing the risks associated with insertional mutagenesis.[5] Moreover, multiple virions can infect a cell and increase the copies of recombinant genes expressed without any viral replication. Safe and easy propagation in tissue culture and efficient in vivo gene transduction make adenoviruses ideal candidates for cancer gene therapy in which only transient expression of a gene is needed.

The efficacy of adenoviruses for gene delivery into brain tumors has been reported by our laboratory and others.[1,2,8] Thus far, however, only iatrogenic malignant brain tumor models have been described. Because adenoviruses can transduce both dividing and nondividing cells, they may also prove beneficial in treating some benign brain tumors that do not respond to conventional therapies. Here, we report 
selective gene delivery into a spontaneous canine meningioma by intraarterial injection of a recombinant adenovirus.

\section{MATERIALS AND METHODS}

\section{Viral Vector}

The adenoviral vector AdHCMVsp1LacZ (AdLacZ) contains an expression cassette encoding the Escherichia coli B-galactosidase gene (LacZ), driven by the cytomegalovirus promoter located upstream from a polyadenylation signal. Because this expression cassette replaces the entire viral E1A and most of the E1B regions (early viral proteins) within the adenovirus, these viral vectors are replication defective. They are able to replicate only in the 293 human embryonic kidney packaging cell line, which expresses the missing E1 proteins. Viral stocks are prepared by vector infection of 293 cells and titered by plaque assays expressed as plaque-forming units (pfu).

\section{Animal Selection}

Approvals from our institutional Research Animal Resource Center and Biological Safety Committee were obtained as part of an ongoing study to evaluate the role of adenoviruses for gene therapy in canine brain tumors. A 14-year-old, 8-kg, spayed female West Highland Terrier presented with new-onset seizures. A diagnosis of olfactory groove meningioma was made on the basis of magnetic resonance imaging of the dog's head (Fig. 1). After a cerebral arteriogram showed an abundant vascular supply to the tumor from the external and internal carotid arteries, the animal was selected for viral injection and informed consent for the procedure was obtained from the dog's owners.

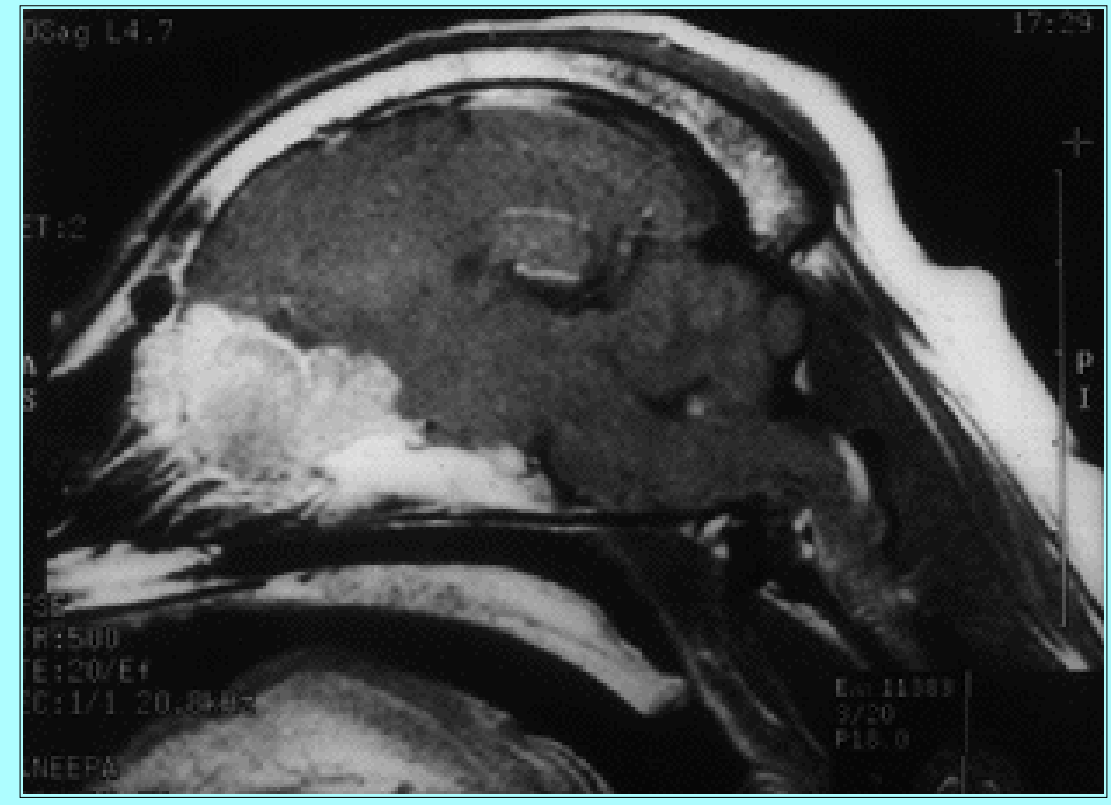

Fig. 1. Contrast-enhanced sagittal magnetic resonance image of the dog's head demonstrating an extraaxial frontal tumor consistent with a meningioma.

\section{Arteriography and Viral Injection}

After general anesthesia was administered, the right femoral artery was catheterized with a No. 4 French angiography catheter. An injection of iohexol (not exceeding $2 \mathrm{ml} / \mathrm{kg}$ total dose) into the left carotid artery revealed that the tumor's blood supply came from both the internal and external left carotid arteries. Approximately $1 \mathrm{ml}$ of AdLacZ ( 2 X $\left.10^{9} \mathrm{pfu} / \mathrm{ml}\right)$ was selectively injected into tumor vessels 
arising from the intracavernous segment of the left internal carotid artery and the left middle meningeal branch of the maxillary artery from the external carotid artery (Fig. 2). The injections were administered as close to the tumor as the catheter size would permit. Because of the dual vascularization and the risk of cerebral ischemia the tumor was not embolized. After the dog was kept 5 days in isolation, a craniotomy was performed for tumor resection. Tumor and peritumoral tissue samples were obtained for routine histopathological investigation and for X-Gal staining. After a 5-day recovery period, the dog returned home with her owners.

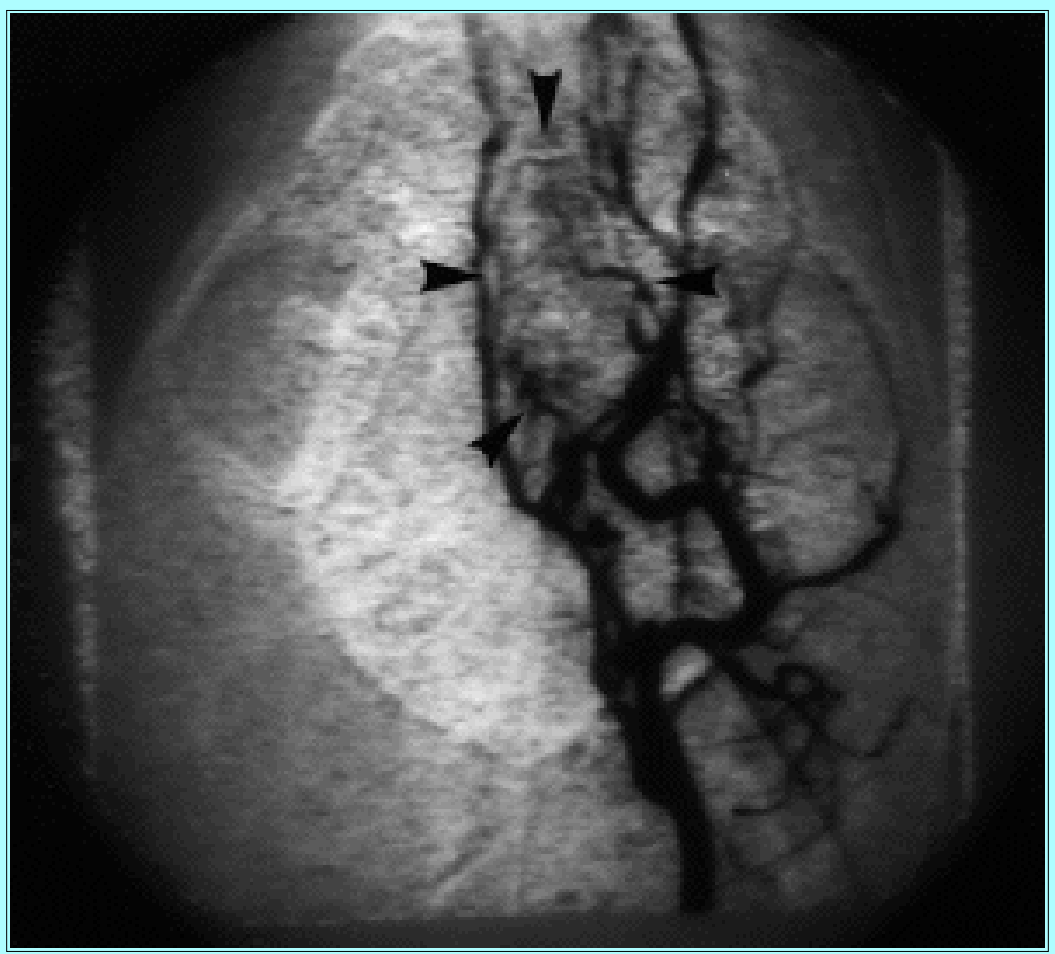

Fig. 2. Cerebral arteriogram, obtained after common carotid injection, showing the tumor blush (arrowheads).

\section{Staining With X-Gal}

Twenty-micrometer-thick frozen sections of tumor and peritumoral tissue were submerged in fixative

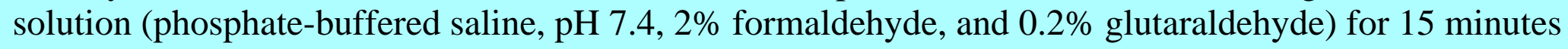
at $4{ }^{\circ} \mathrm{C}$. Samples were then immersed in X-Gal staining solution (phosphate-buffered saline, $5 \mathrm{mM}$ $\mathrm{K}_{4} \mathrm{Fe}(\mathrm{CN})_{6}, 5 \mathrm{mM} \mathrm{K}{ }_{3} \mathrm{Fe}_{3}(\mathrm{CN})_{6}$, and $1 \mathrm{mg} / \mathrm{ml} \mathrm{X-Gal} \mathrm{stain} \mathrm{[GBT,} \mathrm{St.} \mathrm{Louis,} \mathrm{MO])} \mathrm{for} 48$ hours at $37^{\circ} \mathrm{C}$ and then counter-stained with basic fuchsin $(0.25 \%)$. Fragments of tumor and peritumoral tissue were processed in a similar fashion, but were not counterstained.

\section{RESULTS}

\section{Histopathological Findings}

The tumor had the typical histological appearance of a meningioma (Fig. 3). The histopathological characteristics of the peritumoral tissue were consistent with brain, with some areas indicating possible meningioma cell infiltrations, gliosis, and retraction injury. No inflammatory response or lymphocytic infiltration was noted in the meningioma or the peritumoral tissue. 


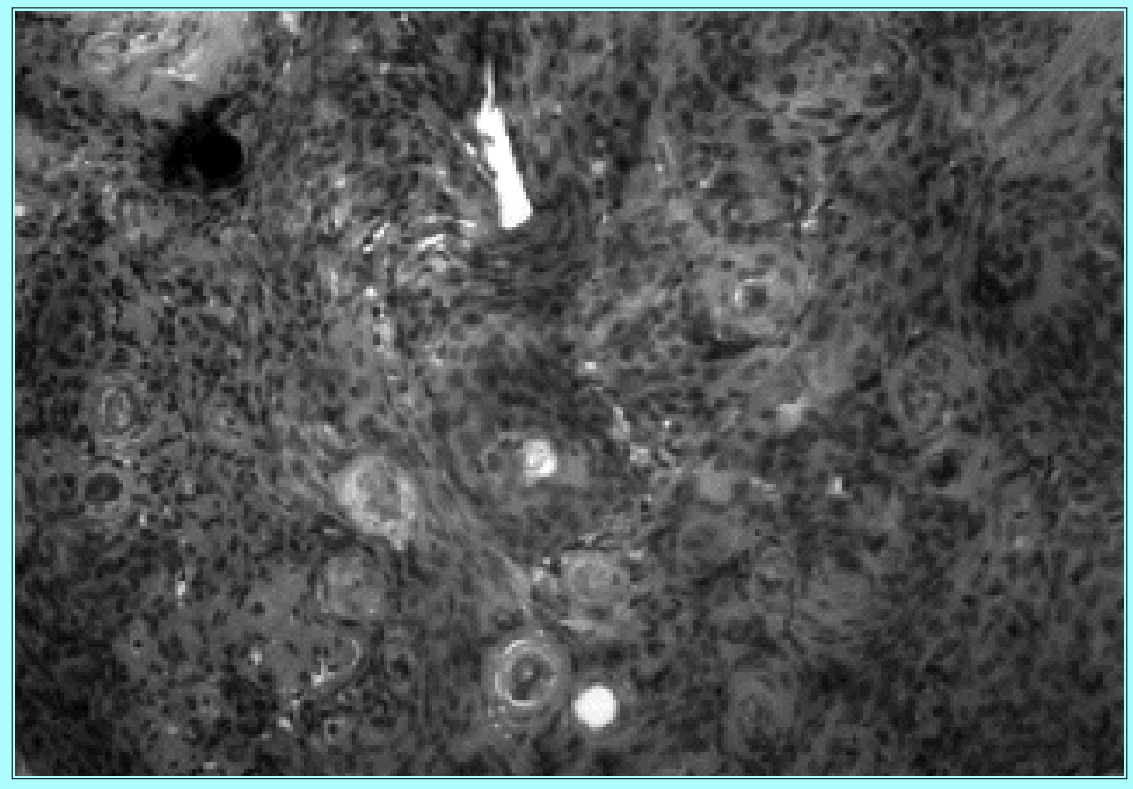

Fig. 3. Photomicrograph of sections of resected tumor demonstrating typical features of a meningioma. H \& E, original magnification X 400.

\section{Viral Gene Delivery}

Successful gene transduction was identified by the blue color reaction of the tissue following X-Gal staining. The upper and lower left panels in Fig. 4 illustrate the significant blue staining of meningioma tumor fragment and cells, indicating efficient gene delivery and expression. In contrast, peritumoral tissue demonstrated a very low level of transduction (Fig. 4 upper and lower right).

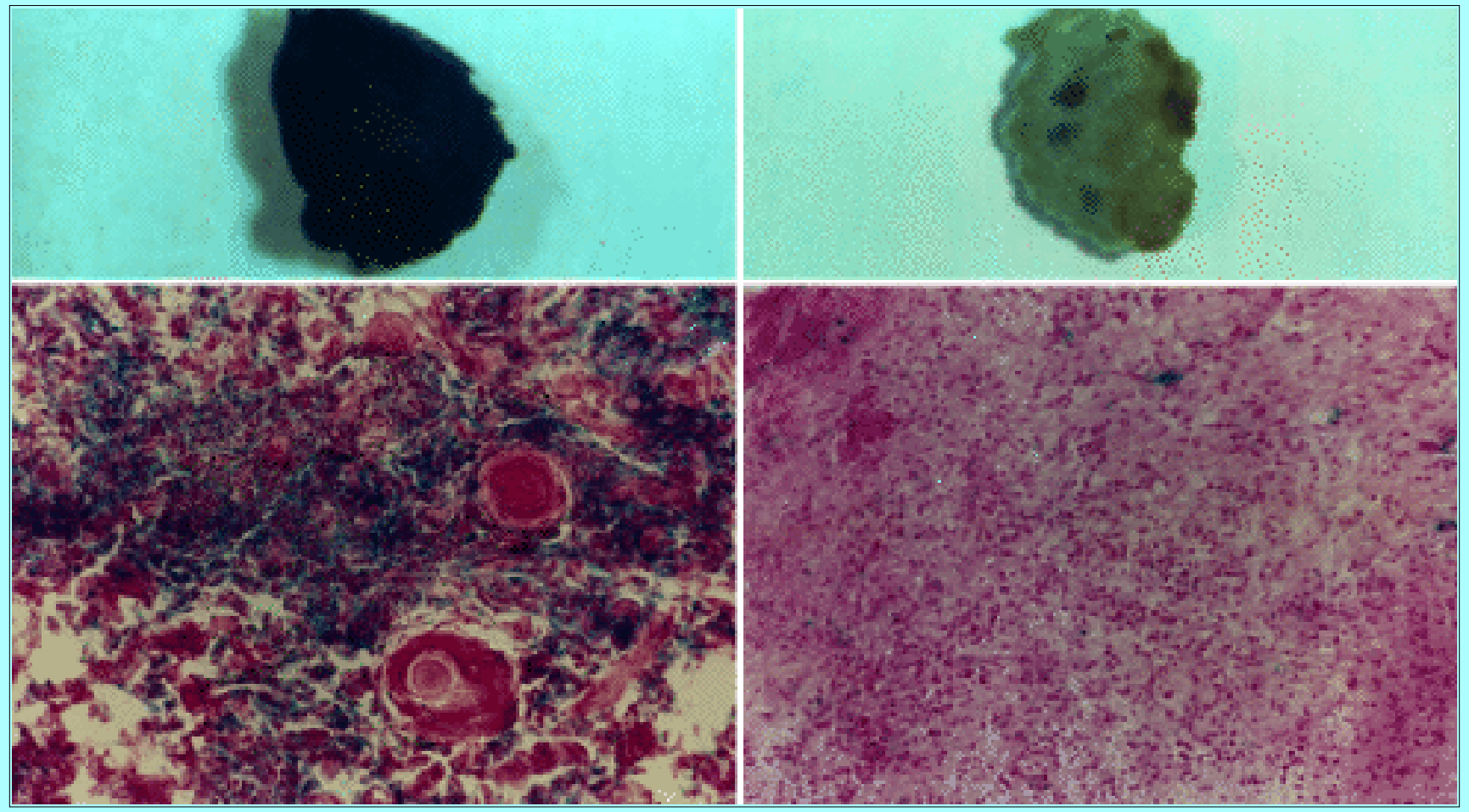

Fig. 4. Tumor fragment (upper left) and frozen section (lower left) demonstrating blue staining, which indicates successful gene expression. Peritumor brain tissue fragment (upper right) and section (lower right) revealing minimal staining. X-Gal staining; tumor fragment size $1 \mathrm{~cm}$; peritumor fragment size $0.5 \mathrm{~cm}$; photomicrographs of tumor and brain tissue sections, original magnification X 200. 


\section{Safety of the Procedure}

Slight stiffness of the right pelvic limb was present for 2 days postcatheterization, but resolved thereafter. No evidence of toxicity from the AdLacZ injection was noted. The pre- and postoperative complete blood count and chemistry panel results were within normal limits, except for the serum alkaline phosphatase level, which was slightly elevated before viral injection and remained unchanged postoperatively. The results of neurological examination remained normal after catheterization and surgery. Anticonvulsant therapy was not required and seizures have not been noted as of 2 months postsurgery.

\section{DISCUSSION}

Meningiomas account for approximately $20 \%$ of primary intracranial neoplasms and are typically curable after complete resection. However, some meningiomas are surgically inaccessible and radiotherapy may only retard their growth. At the present time, there is no effective chemotherapy for the group of meningiomas that do not respond to either surgery or irradiation. Most of these tumors recur locally and have abundant vascular supply, thereby making them good candidates for gene therapy.

In this report, we have demonstrated successful gene delivery into a spontaneously occurring canine meningioma by using recombinant adenoviruses. These vectors were chosen because of their capacity to transduce the nondividing cells found in slowly growing tumors. The discrete vascular supply of this meningioma enabled us to deliver the $\mathbf{B}$-galactosidase gene into the tumor selectively while minimizing virus uptake by surrounding brain tissue. Although we could not assess the extent of virus spread to other organs in this animal, our experience with other dogs undergoing nonselective intracarotid adenovirus injections has been that there is some gene expression in the liver as well as in the brain (unpublished data). With recent developments in endovascular techniques, it may be possible to minimize this virus spread by selective tumor embolization.

Although our study was not specifically designed to address the safety of adenovirus gene therapy, we did not observe any neurological or systemic toxicities in this animal during a 2-month follow-up period. The safety of these vectors for CNS gene therapy has been demonstrated in several reports. Intracarotid viral injections, with or without blood-brain barrier disruption, demonstrated adenoviruses to have low CNS pathogenicity, in contrast with herpes simplex viruses, which lead to varying degrees of necrosis.[7] Moreover, a recent study in which the safety of using adenoviruses in nonhuman primates was evaluated has shown minimal toxicity after intracranial injections of high titers of thymidine kinase-bearing adenovirus without ganciclovir infusion.[4] Although the antigenicity of these vectors has limited their use in systemic gene delivery,[10] it may not be an issue in brain tumor gene therapy because of the partially immunocompromised tumor milieu. Furthermore, second generation adenoviruses with less capacity for viral gene expression and antigenicity may prove to be less cytotoxic in the future.

Unlike gliomas, most meningiomas are compact tumors with abundant gap junctions and may respond more effectively to suicide gene therapy. Intracellular connections have been shown to play a major role in the bystander effect observed in thymidine kinase gene therapy in which cytotoxic ganciclovir metabolites are transported to surrounding nontransfected cells, thus leading to their death.[9] Whether the slow growth of meningiomas prevents their response to thymidine kinase gene therapy will be evaluated in the future. 


\section{Acknowledgment}

Special thanks to Dr. Shahriar Salamat for his assistance in reviewing the histopathological findings in this case.

\section{References}

1. Badie B, Hunt K, Economou JS, et al: Stereotactic delivery of a recombinant adenovirus into a C6 glioma cell line in a rat brain tumor model. Neurosurgery 35:910-916, 1994

2. Chen SH, Shine HD, Goodman JC, et al: Gene therapy for brain tumors: regression of experimental gliomas by adenovirus-mediated gene transfer in vivo. Proc Natl Acad Sci USA 91:3054-3057, 1994

3. Culver KW, Ram Z, Walbridge S, et al: In vivo gene transfer with retroviral vector-producer cells for treatment of experimental brain tumors. Science 256:1550-1552, 1992

4. Goodman JC, Trask GW, Chen SH, et al: Adenoviral-mediated thymidine kinase gene transfer into the primate brain followed by systemic ganciclovir: pathologic, radiologic, and molecular studies. Hum Gene Ther 7:1241-1250, 1996

5. Horowitz M: Adenoviridae and their replication, in Fields BN, Knipe DM (eds): Fields Virology, ed 2. New York: Raven Press, 1990, pp 1679-1740

6. Le Gal La Salle G, Robert JJ, Berrard S, et al: An adenovirus vector for gene transfer into neurons and glia in the brain. Science 259:988-990, 1993

7. Muldoon LL, Nilaver G, Kroll RA, et al: Comparison of intracerebral inoculation and osmotic blood-brain barrier disruption for delivery of adenovirus, herpesvirus, and iron oxide particles to normal rat brain. Am J Pathol 147:1840-1851, 1995

8. Viola JJ, Ram Z, Walbridge S, et al: Adenovirally mediated gene transfer into experimental solid brain tumors and leptomeningeal cancer cells. J Neurosurg 82:70-76, 1995

9. Wu JK, Cano WG, Meylaerts SAG, et al: Bystander tumoricidal effect in the treatment of experimental brain tumors. Neurosurgery 35:1094-1102, 1994

10. Yang Y, Nunes FA, Berencsi K, et al: Cellular immunity to viral antigens limits E1-deleted adenoviruses for gene therapy. Proc Natl Acad Sci USA 91:4407-4411, 1994

Manuscript received March 26, 1997.

Accepted in final form December 16, 1997.

This project was funded in part by Grant No. P30 CA14520 from the National Cancer Institute and funds from the University of Wisconsin Comprehensive Cancer Center to Dr. Badie.

Address reprint requests to: Behnam Badie, M.D., Department of Neurological Surgery, H4/330, Clinical Science Center, 600 Highland Avenue, University of Wisconsin Hospitals and Clinics, Madison, Wisconsin 53792. 